\title{
Analysis of gap times in a two-stage stochastic flowshop with overlapping operations
}

The impact of transfer batching (also referred to as lot splitting) on the performance of flowshops has received widespread attention in the literature. Most papers have emphasized the usefulness of lot splitting in cutting down average flow times, as it enables the overlapping of operations at different stages of the flowshop. However, while most analytical papers have studied deterministic flowshops, an important downside of lot splitting has been overlooked; i.e., the occurrence of idle time between the processing of consecutive sublots belonging to the same process batch (referred to as gap times). Gap times add no value to the product; they merely increase the process batch makespan at the different stages. In deterministic systems, these gap times may be avoided by balancing the processing rates of the different machines in the shop; in stochastic settings, however, they may occur even when the system is perfectly balanced, due to the inherent variability in the setup and processing times. Studying a two-stage flowshop with a single product type, this paper provides insight into the behavior of the gap times, and develops an approximation for the process batch makespan at the second stage in terms of the system characteristics and the lot splitting policy.

\section{Introduction}

In the operations management literature, it is widely acknowledged that batch sizing decisions influence performance measures such as cycle times and work-in-process levels. As these are decisive factors for the responsiveness of any production environment, setting batch sizes in a production system is an important control (e.g., see Hopp and Spearman 2000, Lambrecht et al. 1998, Benjaafar 1996).

When studying the impact of batching decisions in a production environment, one should make a distinction between two types of batches, namely process batches and transfer batches. A process batch (also referred to as a production batch or production lot) is defined as the quantity of a product processed on a machine without interruption by other items (Kropp and Smunt 1990). In multiple-product environments, the use of process batches is often unavoidable due to capacity considerations, as a switch from one product type to the next entails a setup or changeover time, which consumes part of the capacity of the machine. After the setup has been performed, a certain quantity of the product (the process batch size) can be produced. Hence, a process batch can also be defined as the quantity of a product produced between two consecutive setups. 
A transfer batch (or transfer lot) refers to the size of a sublot of the process batch, moved after production on one machine to another operation or machine (Kropp and Smunt 1990). The use of transfer batches is not linked to capacity considerations, but rather to flow considerations. Indeed, it is widely accepted that the use of transfer batch sizes smaller than the process batch size can reduce product flow times by smoothing workflow and minimizing congestion levels (e.g., see Santos and Magazine 1985, Benjaafar 1996, Goldratt and Cox 1984, Hopp et al. 1990 and Umble and Srikanth 1995). This is due to the mechanism of overlapping operations. By allowing transportation of partial batches to a downstream station, this station can already start processing these partial batches while work proceeds at the upstream station, thereby accelerating the progress of work through the production facility (Graves and Kostreva 1986, Jacobs and Bragg 1988, Litchfield and Narasimhan 2000).

There exists a large body of research on the impact of lot splitting in deterministic flowshop environments. A wide range of models has been proposed, depending on the constraints of the environment that one intends to model; e.g., it may be assumed that all sublots have to be of equal size (as in Jacobs and Bragg 1988, Kalir and Sarin 2001), and/or it may be imposed that sublots have to be consistent throughout the system, implying that the same sublot sizes have to be used at each machine (as in Van Nieuwenhuyse and Vandaele 2004). The primary objective of these papers is to determine the optimal lot splitting procedure in order to minimize either the process batch makespan (Chen and Steiner 1996, Chen and Steiner 1998, Cheng et al. 2000), mean transfer batch flow time (Kalir and Sarin 2001, Sen et al. 1998, Bukchin et al. 2002, Van Nieuwenhuyse and Vandaele 2004), or a combination of both (Bukchin and Masin 2004) in such an environment.

While the favorable impact on flow times has received widespread attention, the use of lot splitting also has a downside; it may lead to idle times between the processing of consecutive sublots, belonging to the same process batch. These idle times are referred to as gaps, and are caused by the fact that the setup and processing times at the different stages of the flowshop are not balanced (see Van Nieuwenhuyse and Vandaele, 2004). Hence, it may happen that a downstream stage finishes processing a sublot before the next sublot is available from the upstream stage.

In a deterministic environment, this downside can be avoided by explicitly imposing that a machine may not remain idle between consecutive sublots. This is referred to as the no-idling assumption (e.g., see Baker and Jia 1993, Ramasesh et al. 2000). However, in a 
stochastic flowshop, the occurrence of gaps is a major issue. As setup and processing times at the different stages are variable, gaps may occur even when the average setup and processing times are balanced. The occurrence of these gap times leads to an increase in the makespan of a process batch, without adding value to the product. In fact, gap times may even represent a cost for the system. During a gap time, the server has to be kept operational, such that it is ready for processing the next transfer batch when it arrives (Van Nieuwenhuyse and Vandaele 2004). Depending upon the type of server, this may entail labor and/or machine costs (such as energy costs). From the planner's point of view, it is highly desirable to have an estimate of the extent to which gap times will influence the average makespan of a process batch at a given stage, such that it can be taken into account in the planning system.

The objective of this paper is to develop insights and approximations for the average gap time in a two-stage stochastic flowshop with general setup and processing times. In a stochastic system, the gap time will be a stochastic variable, influenced by the level of variability in the system and the lot splitting policy. Focusing on a flowshop with a single product type, we can derive a lower bound for the average process batch makespan at the second stage, as well as an approximation. The performance of the approximation is tested by means of an extensive simulation experiment, and turns out to be very satisfactory.

To the best of our knowledge, this paper is the first to take a closer look at the occurrence of gap times in a stochastic environment (analytical expressions for the gap times in a deterministic flowshop were derived previously in Van Nieuwenhuyse and Vandaele 2004). In section 2, we first describe the assumptions of our setting. Next, section 3 presents some basic insights for the average gap time, along with a lower bound. As will be shown, the average gap time is analytically intractable. The remainder of the paper then focuses on estimating the average gap time, in order to yield a satisfactory approximation for the average process batch makespan at the second stage. This is done by means of simulation. Section 4 describes the setting of the simulation experiment, and examines a suitable approximation for the average gap time based on the simulation results. Section 5 discusses the resulting approximations for the average process batch makespan, and tests the performance of the approximation versus simulation results, using the lower bound as a benchmark. In section 6, we apply the expressions to an example. Finally, section 7 summarizes the conclusions. 


\section{Notation and assumptions}

As mentioned before, we will consider a two-stage stochastic flowshop with a single product type. The product is produced in process batches of a specific size. Some minor characteristics of the product type (such as color or size) may differ from one process batch to the next, necessitating a setup time at the start of the process batch. The flowshop is assumed to be an open shop; process batches that are launched into the system undergo operations subsequently on the first and second stage, and leave the system after being processed. Setup times as well as unit processing times on both stages are random variables, with an arbitrary probability distribution. The buffers in front of the stages have infinite capacity. For illustrative purposes, Figure 1 shows the progress of a process batch, consisting of 3 sublots, through a two-stage system.

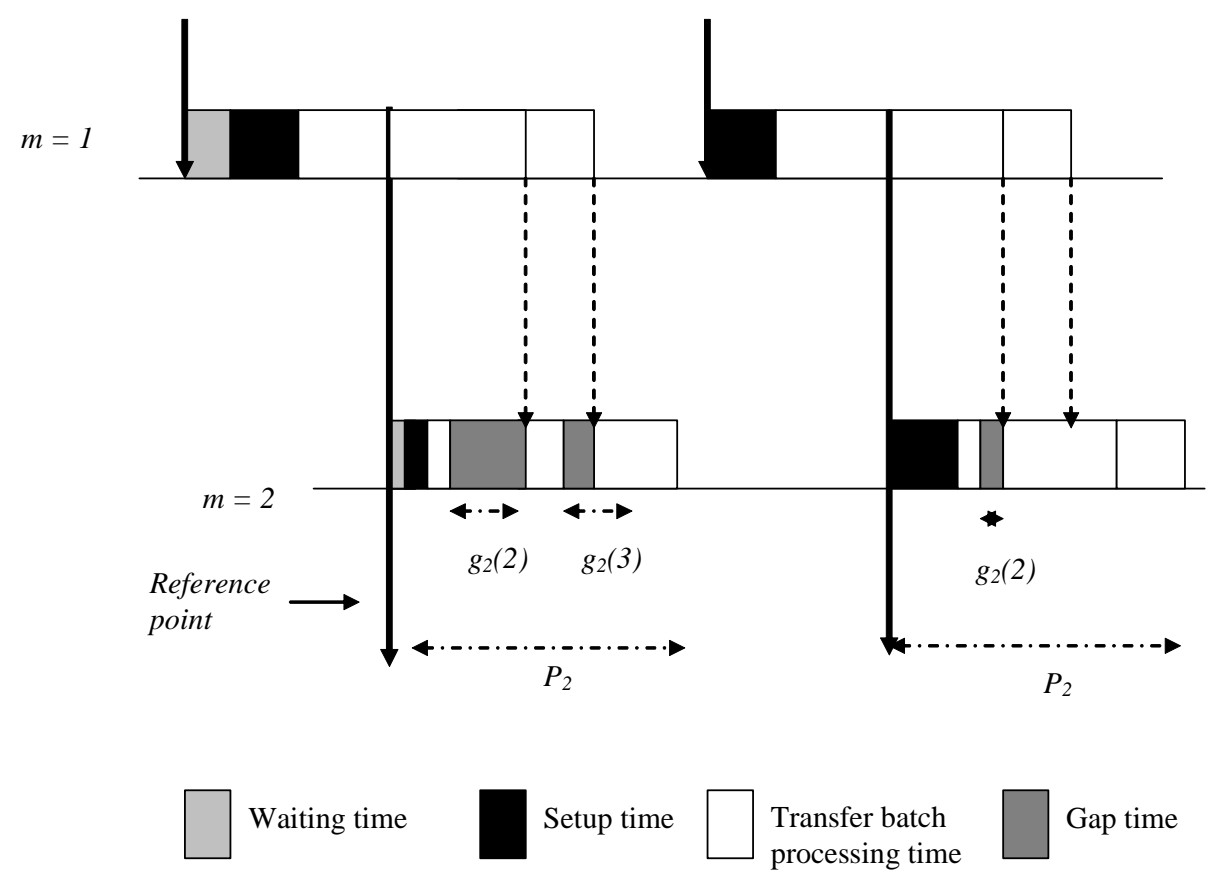

Figure 1. Flowchart of a process batch, consisting of 3 sublots, going through a 2-stage system

Following the assumption of an open system, process batches of size $N$ arrive in front of the first stage. It is assumed that the exact characteristics of a given process batch are not known until that batch has arrived; hence, the setup on the first stage cannot be anticipated. Upon arrival, a process batch may have to wait until the first stage becomes 
available, namely when the stage is still busy processing a previous process batch. As soon as the stage is available, the setup is performed for the current process batch and the units in the batch are processed one by one. After processing, product units are collected to form a transfer batch of size $L(L \leq N)$. As soon as a transfer batch is complete, it is moved to the next server (the transportation time between the two stages is not explicitly modelled). For simplicity as well as practical reasons, $L$ is supposed to be a divisor of $N$, such that a single process batch is split into an integer number $(T)$ of transfer batches $(T=N / L)$.

It is important to note that, in our setting, the setup time on stage two is an attached setup (Chen and Steiner 1998, Potts and Kovalyov 2000), meaning that it can only start when the first transfer batch of the involved process batch has arrived in the input buffer of the second stage ${ }^{1}$. Hence, the first transfer batch of a process batch acts as a flag (Smunt et al. 1996); its arrival in front of the second stage authorizes the start of the setup, thereby causing the operations on stage two to partly overlap with the operations on stage one. Upon arrival at the second stage, the flag may have to wait until the server becomes idle, namely when the server is still processing a transfer batch belonging to the previous process batch; this waiting time will be denoted by $W_{2}$. Next, the setup for the process batch is performed and the flag is processed. The remaining sublots are processed as soon as they are finished on stage one, provided that stage two is available at that moment; otherwise, they will wait in the input buffer of the second stage. After stage two, the transfer batches belonging to the same process batch are regrouped for shipment.

The setup time required at the start of every process batch at a stage $m(m=1,2)$ will be denoted by $S_{m}(\geq 0)$. While some papers in the literature consider setup times to be necessary at the start of every transfer batch (e.g., see Bukchin et al. 2002), we consider setups only at the start of a process batch. It is assumed that setup times for the separate transfer batches, e.g., for mounting the parts on the machine, are either negligible or included in the process time.

The processing time for a transfer batch $i$ on stage $m$ is denoted by $X_{m, i}$ with $i$ ranging from 1 to $T$. In our two-stage setting, each of the stages is assumed to be a capacity server. This means that the transfer batch processing time on each of the stages is dependent on the transfer batch size, and hence, $X_{m, i}$ can be expressed as the sum of $L$ unit processing

\footnotetext{
${ }^{1}$ This assumption facilitates a future extension of the model towards a job shop setting with multiple product types. In a job shop setting, every server will be ignorant of the exact characteristics of the next process batch to serve, until the moment at which the batch has effectively arrived in its input buffer.
} 
times on server $m$, for any arbitrary $i$ :

$$
X_{m, i}=\sum_{j=(i-1) L+1}^{i L} x_{m, j}
$$

In this expression, $x_{m, j}$ refers to the processing time of the $j$ th product unit in the process batch. The makespan of a process batch at stage 2 is denoted by $P_{2}$; it is a stochastic variable, consisting of the setup time at stage $2\left(S_{2}\right)$, the individual transfer batch processing times $\left(X_{2, i}\right)$, and the total gap time $\left(G_{2}\right)$. The total gap time is defined as the sum of the idle times, occurring between the processing of two consecutive sublots belonging to the same process batch. Consequently, it consists of a number of partial gaps $g_{2}(i)$ ( where $i$ denotes the number of the transfer batch which immediately follows the partial gap):

$$
G_{2}=\sum_{i=2}^{T} g_{2}(i)
$$

It is clear that a gap can never occur in front of the flag (hence, $g_{2}(1)=0$ ), due to the attached setup at stage 2 . It is useful to note that in our definition, a gap time refers to an idle time occurring between the processing of consecutive sublots from the same process batch. Hence, it does not include any idle time that may occur between the processing of two distinct process batches.

\section{Basic insights and lower bound for the average gap time}

For studying the total gap time $G_{2}$, we only have to take into account how the system behaves after the so-called reference point; i.e., the point in time when the flag is finished on stage 1 and moved to the next machine (as depicted in Figure 1). As mentioned above, $G_{2}$ is given by

$$
G_{2}=\sum_{i=2}^{T} g_{2}(i)
$$

A partial gap $g_{2}(i)$ can be written as

$$
g_{2}(i)=\max [\underbrace{\sum_{k=2}^{i} X_{1, k}-\left(W_{2}+S_{2}+\sum_{j=1}^{i-1}\left[X_{2, j}+g_{2}(j)\right]\right)}_{V(i)}, 0] \quad(i=2, \ldots, T)
$$


From this expression, we can derive that the probability distribution of a partial gap time $g_{2}(i)$ will have a zero-inflated shape. The probability that $g_{2}(i)$ equals zero is given by the probability that $V(i)$ is smaller than or equal to zero. For $g_{2}(i)>0$, the density function of $g_{2}(i)$ is equal to the density function of $V(i)$.

Based upon expressions (1) and (2), the total gap $G_{2}$ can be written as

$$
G_{2}=\sum_{i=2}^{T} \max [\underbrace{\sum_{k=2}^{i} X_{1, k}-\left(W_{2}+S_{2}+\sum_{j=1}^{i-1}\left[X_{2, j}+g_{2}(j)\right]\right)}_{V(i)}, 0]
$$

which can be rewritten as

$$
G_{2}=\max [Z(2), \ldots, Z(T), 0]
$$

with $Z(i)=\sum_{k=2}^{i} X_{1, k}-\left(W_{2}+S_{2}+\sum_{j=1}^{i-1} X_{2, j}\right)$ for $(i=2, \ldots, T)$. As this expression shows, the total gap is a random variable consisting of the maximum of $T-1$ correlated random variables $Z(t)$ (with $t=2, \ldots, T$ ) and a constant (zero). A formal proof for expression (4) is presented in Appendix 1.

Based on expression (4), we can write the following for the average of the total gap:

$$
E\left[G_{2}\right]=E[\max [Z(2), \ldots, Z(T), 0]]
$$

As the exact distribution of each of the variables $Z(t)$ is unknown in a general setting, $E\left[G_{2}\right]$ is analytically intractable. We may, however, derive a tractable lower bound. Indeed, based on Jensen's inequality (e.g., see Ross 1996), we know that

$$
E[\max [Z(2), \ldots, Z(T), 0]] \geq \max [E[Z(2)], \ldots, E[Z(T)], 0]
$$

such that a lower bound is given by

$$
E\left[G_{2}\right]_{L B}=\max [E[Z(2)], \ldots, E[Z(T)], 0]
$$

In section 4 , we develop an approximation for $E\left[G_{2}\right]$. When testing the appropriateness of this approximation (see section 5), the performance of the lower bound will be used as a benchmark. 


\section{Approximation for the average gap time}

\subsection{Simulation experiment}

As mentioned above, an analysis of the average gap time $E\left[G_{2}\right]$ can be done by considering the system behavior after the reference point (see Figure 1). In view of this analysis, a simulation experiment was designed using the following variables as factors:

$T$ : number of transfer batches per process batch;

$L$ : transfer batch size;

$E\left(X_{1}\right)$ : average processing time per transfer batch on stage 1;

$E\left(X_{2}\right) / E\left(X_{1}\right)$ : ratio of the average processing time per transfer batch on stage 2 versus the average processing time per transfer batch on stage 1 ;

$E\left(S_{2}\right) / E\left(X_{1}\right)$ : ratio of the average setup time per process batch on stage 2 versus

the average processing time per transfer batch on stage 1;

$c_{X_{1}}^{2}$ : squared coefficient of variation $(\mathrm{SCV})$ of the processing time per transfer batch on stage 1 ;

$c_{X_{2}}^{2}$ : SCV of the processing time per transfer batch on stage 2 ;

$c_{S_{2}}^{2}:$ SCV of the setup time on stage 2 ;

probability distributions used for $S_{2}, X_{1}$, and $X_{2}$;

$\rho_{1}$ : utilization rate of stage 1 .

The average and variance of $S_{1}$ were not used as factors in the simulation model, as $S_{1}$ occurs before the reference point. Hence, we have reason to believe that the average and the SCV of $S_{1}$ only have a minor impact on the outcome for $E\left[G_{2}\right]$ in the stochastic model. Both parameters can at best have an indirect impact, as they may influence $E\left[W_{2}\right]$, which on its turn affects $E\left[G_{2}\right]$ (as evident from expression (4)). Given its minor importance, $S_{1}$ was arbitrarily fixed at 15 time units (deterministic) in the experiment.

Table 1 gives an overview of the values that were used in the simulation for the different factors. Combining all factors at all values, this design resulted in 23,328 runs. The length of each run was fixed at 100,000 process batches, the first 20,000 of which were considered as warm-up period.

The interarrival times of process batches in front of stage 1 are deterministic, and controlled in order to yield the desired utilization rate $\rho_{1}$. Note that the combination of certain

values of $\rho_{1}, \frac{E\left(X_{2}\right)}{E\left(X_{1}\right)}$ and $\frac{E\left(S_{2}\right)}{E\left(X_{1}\right)}$ yields a utilization rate higher than or equal to 1 on stage 2 ; in that case, the system is infeasible and the corresponding run is omitted. 
Table 1. Input factors and values for the simulation experiment

\begin{tabular}{l|ll} 
Factor & Values & Number of values \\
\hline \hline$T=\frac{N}{L}$ & $10,15,20$ & 3 \\
$L$ & 1 & 1 \\
$E\left(X_{1}\right)$ & 10 & 1 \\
$\frac{E\left(X_{2}\right)}{E\left(X_{1}\right)}$ & $0.2,0.5,0.8,1,1.2,1.5,1.8,2$ & 8 \\
$\frac{E\left(S_{2}\right)}{E\left(X_{1}\right)}$ & $\frac{1}{3}, \frac{1}{2}, 1, \frac{3}{2}$ & 4 \\
$c_{X_{1}}^{2}$ & $0.3,0.6,0.9$ & 3 \\
$c_{S_{2}}^{2}$ & $0.3,0.6,0.9$ & 3 \\
$c_{X_{2}}^{2}$ & $0.3,0.6,0.9$ & 3 \\
probability distribution & gamma, beta, lognormal & 3 \\
$\rho_{1}$ & $0.4,0.7,0.95$ & 3 \\
\hline
\end{tabular}

\subsection{Approximation for the density function of the total gap time $\mathrm{G}_{2}$}

By plotting the observed frequency distribution of $G_{2}$ for different simulation runs of the experiment, we could draw important insights about the behavior of the gap time in terms of the difference in average processing rates, and the difference in processing time variability on the two stages.

Figure 2 shows the histograms (gamma, beta and lognormal) for $G_{2}$ when $T=20$, $\rho_{1}=0.4, \frac{E\left(S_{2}\right)}{E\left(X_{1}\right)}=0.5$, and $c_{X_{1}}^{2}=c_{S_{2}}^{2}=c_{X_{2}}^{2}=0.3$. The figure in the top pane refers to the setting where $\frac{E\left(X_{2}\right)}{E\left(X_{1}\right)}=0.2$, so where stage 2 has a significantly higher processing rate than stage 1. As expected, the total gap time observed in this setting is always larger than zero. The probability distribution seems to be strikingly close to normal. Moreover, the histograms of the beta, gamma and lognormal distributions almost coincide.

The figure in the bottom pane refers to the setting where $\frac{E\left(X_{2}\right)}{E\left(X_{1}\right)}=0.8$, so where the two processing rates are almost equal. This histogram is zero-inflated, showing a sharp peak at $G_{2}=0$. This is not surprising; it is intuitively clear that, as the ratio of $E\left(X_{2}\right)$ to $E\left(X_{1}\right)$ increases, the observed total gap time $G_{2}$ will more frequently equal zero. More importantly, the positive values of $G_{2}$ appear to be close to a normal distribution. Note that the variability of this distribution is considerably higher than the variability observed in the top pane.

The histograms in Figure 3 give further insight into the impact of variability. They show the 

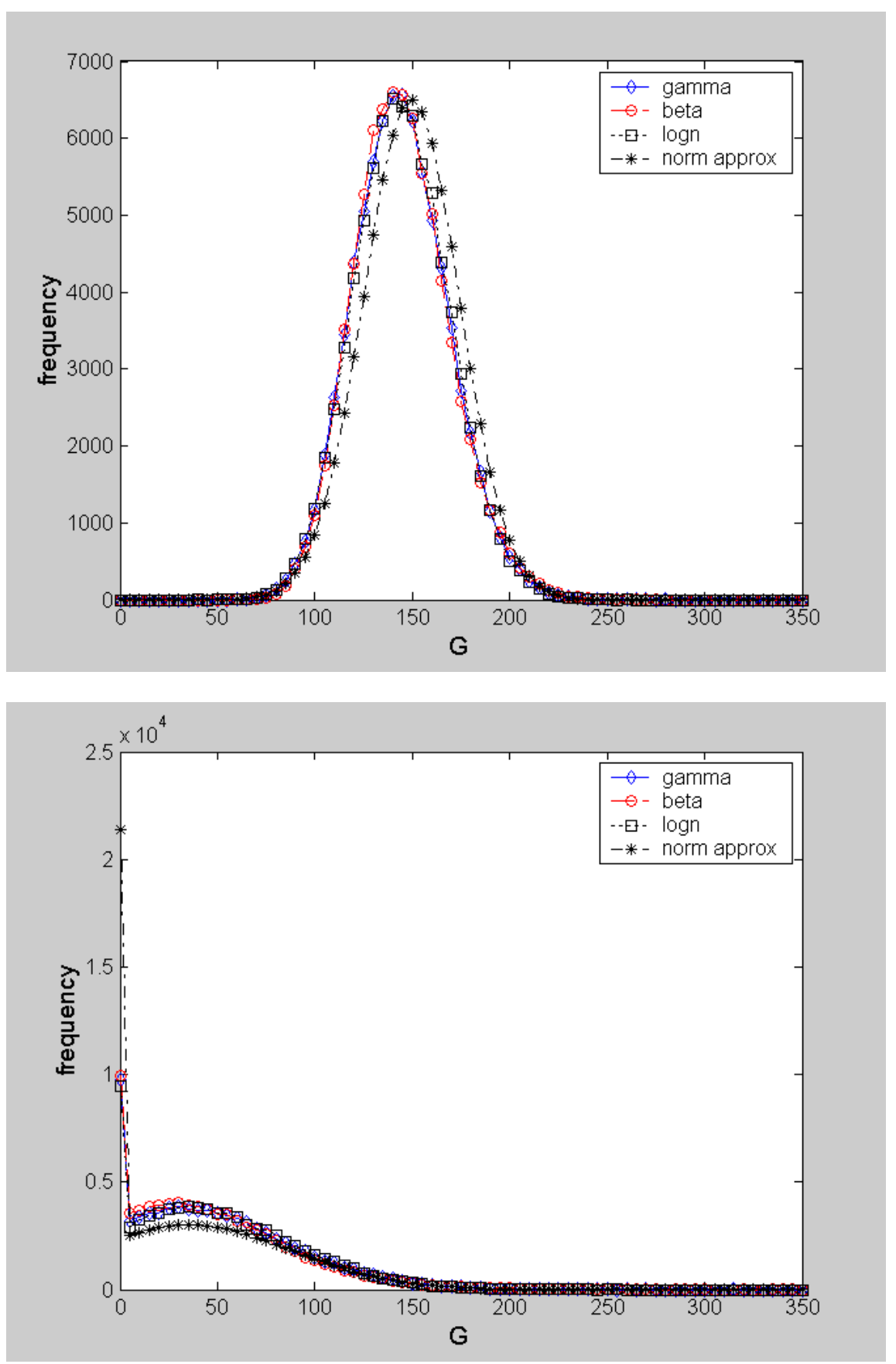

Figure 2. Sample histogram for $G_{2}$ when $\frac{E\left(X_{2}\right)}{E\left(X_{1}\right)}=0.2$ (top pane), and $\frac{E\left(X_{2}\right)}{E\left(X_{1}\right)}=0.8$ (bottom pane), when $c_{X_{1}}^{2}=c_{S_{2}}^{2}=c_{X_{2}}^{2}=0.3$. 
observed frequency distribution of $G_{2}$ for the same settings as Figure 2, but with a higher SCV for setup and processing times $\left(c_{X}^{2}=c_{S_{2}}^{2}=c_{X_{2}}^{2}=0.9\right)$. Apparently, even in situations when the processing and setup times are highly variable, the density of $G_{2}$ is still close to normal. A comparison of the histograms in the top panes of Figures 2 and 3 reveals that a higher variability in setup and processing times leads to a higher variability in $G_{2}$.

Histograms for other simulation settings yielded similar results. The histograms lead us to conjecture that the density function of $G_{2}$ can be reasonably approximated by

$$
G_{2}=\max [V, 0], \quad \text { with } V \simeq N\left(m_{V}, \sigma_{V}^{2}\right)
$$

Hence, $G_{2}$ can be written as the maximum of a normal variable $V$ and 0 . Following this argument, the probability mass observed at $G_{2}=0$ in settings with high values of $\frac{E\left(X_{2}\right)}{E\left(X_{1}\right)}$ can be approximated by the cumulative distribution function of $V$ at 0 :

$$
\operatorname{prob}\left(G_{2}=0\right)=\int_{-\infty}^{0} f_{V}(x) d x=F_{V}(0)
$$

where $f_{V}(x)$ and $F_{V}(x)$ denote respectively the (normal) density function and the (normal) cumulative distribution function of $V$.

The issue now is to determine $m_{V}$ and $\sigma_{V}^{2}$, the mean and variance of this underlying normal distribution. Based upon further study of the histograms, the following candidates can be proposed (Van Nieuwenhuyse 2004):

$$
\begin{aligned}
m_{V} & =(T-1)\left[E\left(X_{1}\right)-E\left(X_{2}\right)\right]-E\left(S_{2}\right) \\
\sigma_{V}^{2} & =(T-1)\left[\operatorname{Var}\left(X_{1}\right)+\operatorname{Var}\left(X_{2}\right)\right]+\operatorname{Var}\left(S_{2}\right)
\end{aligned}
$$

For illustrative purposes, the density function of the corresponding (zero-inflated) normal distribution, with $m_{V}$ and $\sigma_{V}^{2}$ given as in expression (6), is depicted in Figures 2 and 3, revealing that this approximation provides a close fit.

It is particularly useful to confront the proposed approximation for $G_{2}$ with the theoretically exact expression derived above (expression (4)). As mentioned before, this expression shows that the total gap time $G_{2}$ is a random variable given by the maximum of $T-1$ correlated random variables (where $T$ refers to the number of sublots), and a constant (zero). Using 

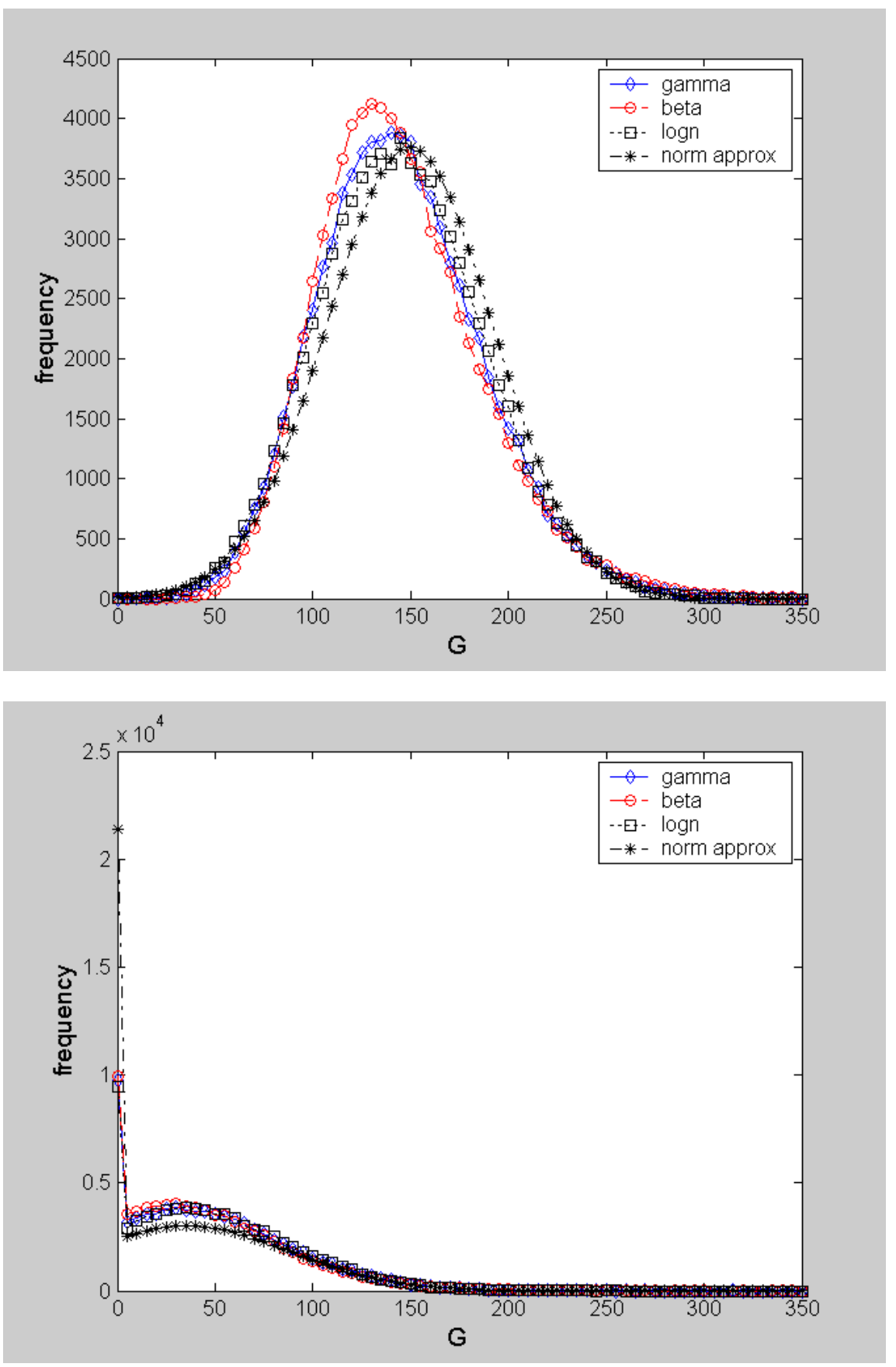

Figure 3. Sample histogram for $G_{2}$ when $\frac{E\left(X_{2}\right)}{E\left(X_{1}\right)}=0.2$ (top pane), and $\frac{E\left(X_{2}\right)}{E\left(X_{1}\right)}=0.8$ (bottom pane), when $c_{X}^{2}=c_{S_{2}}^{2}=c_{X_{2}}^{2}=0.9$. 
the parameters for $m_{V}$ and $\sigma_{V}^{2}$ proposed in (6), $V$ is de facto approximated by

$$
\begin{aligned}
V & =Z(T)+W_{2} \\
& =\sum_{k=2}^{T} X_{1, k}-\left(S_{2}+\sum_{j=1}^{T-1} X_{2, j}\right)
\end{aligned}
$$

Hence, our approximation implicitly makes three simplifying assumptions:

\section{The impact of $\mathbf{W}_{2}$ on $\mathrm{G}_{2}$ is ignored:}

Indeed, expression (4) (which is analytically exact) reveals that there is a negative relationship between the waiting time of the flag in front of stage $2\left(W_{2}\right)$ and the gap time occurring on stage $2\left(G_{2}\right)$. As we ignore this relationship, our approximation will tend to overestimate of the average gap time in cases where stage 2 is highly utilized. However, we do not expect this simplification to lead to significant errors on the estimation of the process batch makespan on stage 2. Large values for $W_{2}$ are bound to occur when the processing rate of stage 2 is relatively low, implying that gap times will constitute a very small (or even negligible) part of the process batch makespan when compared to the actual processing time.

\section{The impact of $Z(2)$ to $Z(T-1)$ on $G_{2}$ is ignored:}

Expression (4) reveals that it suffices to have a positive value for any arbitrary $Z(i)$ $(i=2, \ldots, T)$ in order to have a positive gap time on stage 2 . In our approximation, we only take into account the impact of $Z(T)$. This simplification may negatively impact the performance of our approximation in systems where $E\left(X_{1}\right)$ is close to $E\left(X_{2}\right)$, or larger than $E\left(X_{2}\right)$. In such systems, there is a higher probability for $Z(T)$ to be negative while one of the other $Z(i)(i=2, \ldots, T-1)$ is positive. Hence, we can expect the approximation to underestimate the probability of a gap time occurring for this type of systems. Conversely, we can expect that the simplification will have little effect on the performance of our approximation, as long as $E\left(X_{1}\right)$ is significantly larger than $E\left(X_{2}\right)$; i.e., when the line is unbalanced, with the bottleneck on the first machine.

\section{We assume that $\mathrm{Z}(\mathbf{T})+\mathbf{W}_{2}$ can be reasonably approximated by a normal probability distribution:}

For high values of $T$, we can indeed expect that the density function of $Z(T)+W_{2}$ will be close to normal by virtue of the central limit theorem. As the processing times $X_{1, k}$ on stage 1 are IID distributed, $\sum_{k=2}^{T} X_{1, k}$ will approach a normal distribution with mean 
$m_{1}=(T-1) E\left[X_{1}\right]$ and variance $\sigma_{1}^{2}=(T-1) \operatorname{Var}\left[X_{1}\right]$ for high values of $T$. Similarly, $\sum_{k=2}^{T} X_{2, k}$ will also approach a normal distribution with mean $m_{2}=(T-1) E\left[X_{2}\right]$ and variance $\sigma_{2}^{2}=(T-1) \operatorname{Var}\left[X_{2}\right]$. As the difference of two normal distributions is again a normal distribution (e.g., see Blumenfeld 2001), we can expect that, for high values of $T, \sum_{k=2}^{T} X_{1, k}-\sum_{k=2}^{T} X_{2, k}$ will be normally distributed with mean $m=m_{1}-m_{2}$ and variance $\sigma^{2}=\sigma_{1}^{2}+\sigma_{2}^{2}$. The only disturbing factor is the presence of $S_{2}$ in $Z(T)$; however, if $S_{2}$ is relatively small compared to $\sum_{k=2}^{T} X_{1, k}$ and $\sum_{k=2}^{T} X_{2, k}$, we can expect its impact to be negligible.

\subsection{Approximation for $\mathrm{E}\left[\mathrm{G}_{2}\right]$}

Using $m_{V}$ and $\sigma_{V}^{2}$ as in expression (6), and assuming a normal distribution, the approximation for $E\left[G_{2}\right]$ is given by

$$
\begin{aligned}
E\left[G_{2}\right]_{a p p} & \approx \int_{0}^{+\infty} x\left[\frac{1}{\sqrt{2 \pi \sigma_{V}^{2}}} \operatorname{Exp}\left\{\frac{-\left(x-m_{V}\right)^{2}}{2 \sigma_{V}^{2}}\right\}\right] d x \\
& =\left[\frac{-\exp \left\{\frac{-\left(x-m_{V}\right)^{2}}{2 \sigma_{V}^{2}}\right\} \sigma_{V}^{2}+m_{V} \sqrt{\frac{\pi}{2}} \sigma_{V} \operatorname{Erf}\left[\frac{x-m_{V}}{\sqrt{2} \sigma_{V}}\right]_{0}^{+\infty}}{\sqrt{2 \pi} \sigma_{V}}\right. \\
& =\frac{m_{V}}{2}+\frac{\operatorname{Exp}\left\{\frac{-m_{V}^{2}}{2 \sigma_{V}^{2}}\right\} \sigma_{V}^{2}}{\sqrt{2 \pi} \sigma_{V}}+\frac{m_{V}}{2} \operatorname{Erf}\left[\frac{m_{V}}{\sqrt{2} \sigma_{V}}\right]
\end{aligned}
$$

in which $\operatorname{Erf}[z]$ refers to the error function

$$
\operatorname{Erf}[z]=\frac{2}{\sqrt{\pi}} \int_{0}^{z} \exp \left\{-t^{2}\right\} d t
$$

Note that expression (7) satisfies the lower bound defined in expression (5). Indeed, we can rewrite expression (7) as follows:

$$
\begin{aligned}
E\left[G_{2}\right]_{a p p} & \approx m_{V}-\underbrace{\int_{-\infty}^{0} x\left[\frac{1}{\sqrt{2 \pi \sigma_{V}^{2}}} \operatorname{Exp}\left\{\frac{-\left(x-m_{V}\right)^{2}}{2 \sigma_{V}^{2}}\right\}\right] d x}_{<0} \\
& >m_{V}
\end{aligned}
$$

As $m_{V}=E\left[Z(T)+W_{2}\right]=E[Z(T)]+E\left[W_{2}\right]$, we then have

$$
E[Z(T)]<m_{V}<E\left[G_{2}\right]_{a p p}
$$

which proves that the approximation satisfies the lower bound. 


\section{Performance of the approximation for the average process batch makespan}

Using expression (7) for $E\left[G_{2}\right]_{a p p}$, we can now develop an approximation for $E\left[P_{2}\right]$ :

$$
E\left[P_{2}\right]_{a p p} \approx E\left(S_{2}\right)+T E\left(X_{2}\right)+\frac{m_{V}}{2}+\frac{\operatorname{Exp}\left\{\frac{-m_{V}^{2}}{2 \sigma_{V}^{2}}\right\} \sigma_{V}^{2}}{\sqrt{2 \pi} \sigma_{V}}+\frac{m_{V}}{2} \operatorname{Erf}\left[\frac{m_{V}}{\sqrt{2} \sigma_{V}}\right]
$$

Moreover, the lower bound on $E\left[G_{2}\right]$ in expression (5) gives us the following lower bound on $E\left[P_{2}\right]:$

$$
E\left[P_{2}\right]_{L B}=E\left(S_{2}\right)+T E\left(X_{2}\right)+\max [E[Z(2)], \ldots, E[Z(T)], 0]
$$

The performance of this approximation was tested versus the simulation results of the experiment, by determining the relative error $\varepsilon$ of the approximation $\left(E\left[P_{2}\right]_{\text {app }}\right)$ to the observed estimate of $E\left[P_{2}\right]$ in the simulations $\left(E\left[P_{2}\right]_{\text {sim }}\right)$ :

$$
\varepsilon=\frac{\left(E\left[P_{2}\right]_{a p p}-E\left[P_{2}\right]_{s i m}\right)}{E\left[P_{2}\right]_{s i m}}
$$

and the absolute values of these relative errors:

$$
\varepsilon_{a b s}=\left|\frac{\left(E\left[P_{2}\right]_{a p p}-E\left[P_{2}\right]_{s i m}\right)}{E\left[P_{2}\right]_{s i m}}\right|
$$

As a benchmark, we also determined the performance of the lower bound. Table 2 gives an overview of some key statistics for the relative errors incurred by the approximation, along with those of the lower bound.

Table 2. Summary statistics for the relative errors of $E\left[P_{2}\right]_{\text {app }}$ and $E\left[P_{2}\right]_{L B}$ compared to $E\left[P_{2}\right]_{s i m}$ (in percent)

\begin{tabular}{l|ll} 
& $E\left[P_{2}\right]_{a p p}$ & $E\left[P_{2}\right]_{L B}$ \\
\hline \hline average $\varepsilon$ & -1.54 & -4.36 \\
stdev $\varepsilon$ & 1.62 & 4.13 \\
median $\varepsilon$ & -1.42 & -2.93 \\
$5 \%$ percentile $\varepsilon$ & -4.23 & -12.47 \\
$95 \%$ percentile $\varepsilon$ & 0.76 & -0.07 \\
$\max \varepsilon$ & 5.53 & 0.00004 \\
$\min \varepsilon$ & -5.41 & -17.88 \\
average $\varepsilon_{\text {abs }}$ & 1.78 & 4.36 \\
stdev $\varepsilon_{\text {abs }}$ & 1.36 & 4.13 \\
\hline
\end{tabular}



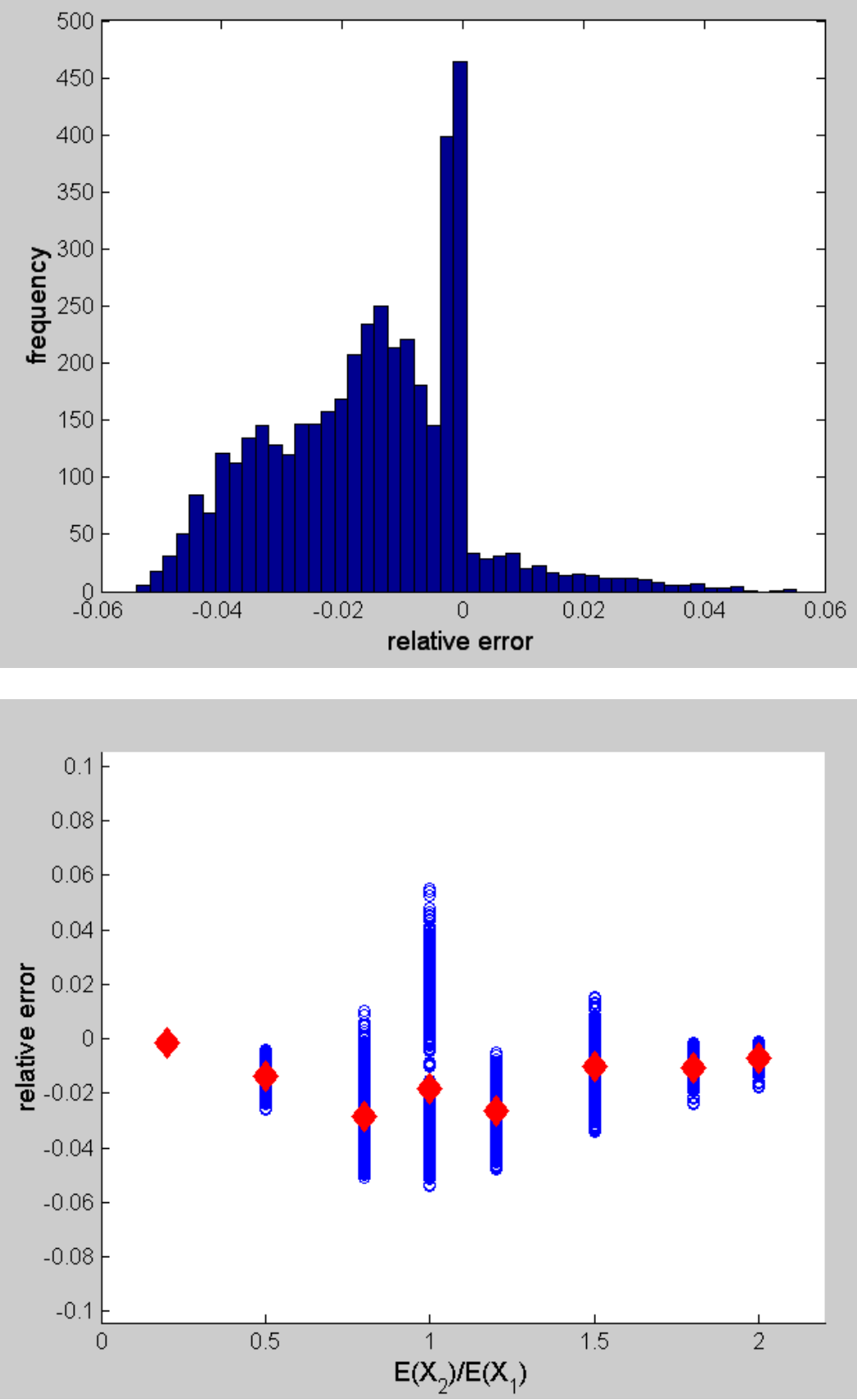

Figure 4. Histogram and scatterplot of the relative errors of $E\left[P_{2}\right]_{a p p}$ versus $E\left[P_{2}\right]_{\text {sim }}$ 
The table reveals that the range of relative errors on the approximation is very small, and that the overall performance of the approximation is satisfactory. In general, $E\left[P_{2}\right]_{a p p}$ tends to underestimate $E\left[P_{2}\right]_{\text {sim }}$. This is evident from the histogram of the relative errors, shown in the top pane of Figure 4. The histogram also shows that the observed frequency of the relative errors is largest at the bin containing $\varepsilon=0$, which is an encouraging characteristic. The tendency to underestimate is not surprising; as mentioned before, $G_{2}$ is theoretically given by the maximum of $(T-1)$ correlated random variables $(Z(2), \ldots, Z(T))$, and zero (see expression (4)). Hence, $G_{2}$ will be positive whenever one of the $Z(i)(i=2, \ldots, T-1)$ is positive; our approximation however only reflects positive values for $Z(T)$, as we have neglected the impact of $Z(2)$ to $Z(T-1)$ (see assumption 2 above).

As expected, the precision of the approximation decreases slightly when the line is closely balanced $\left(E\left(X_{2}\right)\right.$ close to $\left.E\left(X_{1}\right)\right)$. This is confirmed by the bottom pane of Figure 4 , which shows a scatterplot of the relative errors of the experiment in terms of $\frac{E\left(X_{2}\right)}{E\left(X_{1}\right)}$. However, this observation does not undercut the power of the model; on the contrary, it is exactly in these settings that the model proves its full usefulness. As mentioned in the introduction, it is precisely in the case of balanced lines that gap times are hard to analyze, because they are primarily the consequence of the setup and processing time variability in the system. In balanced settings, the lower bound $E\left[G_{2}\right]_{L B}$ will perform particularly poor, as it completely fails to take into account the impact of these variabilities.

This is illustrated in the top pane of Figure 5, which shows the average value of $E\left(G_{2}\right)_{\text {sim }}$ in terms of $\frac{E\left(X_{2}\right)}{E\left(X_{1}\right)}$ for the experiment, along with the average value of $E\left(G_{2}\right)_{L B}$. For unbalanced lines, the performance of $E\left(G_{2}\right)_{L B}$ is rather good, as the occurrence of a gap time in these settings will be largely determined by the difference between the processing rates. However, $E\left(G_{2}\right)_{L B}$ falls to zero as soon as $E\left(X_{2}\right)=E\left(X_{1}\right)$, while in reality, $E\left(G_{2}\right)_{\text {sim }}$ continues to gradually decrease as $\frac{E\left(X_{2}\right)}{E\left(X_{1}\right)}$ increases, approaching zero but never actually reaching zero. Hence, $E\left(G_{2}\right)_{L B}$ seriously underestimates the simulated $E\left(G_{2}\right)$ for $\frac{E\left(X_{2}\right)}{E\left(X_{1}\right)}$ close to 1 .

The structure of the approximation ensures that $E\left(G_{2}\right)_{a p p}$ remains positive and only gradually drops to zero as $E\left(X_{2}\right)$ becomes significantly larger than $E\left(X_{1}\right)$. Indeed, the bottom pane of figure 5 shows that $E\left(G_{2}\right)_{\text {app }}$ follows the $E\left(G_{2}\right)_{\text {sim }}$ very closely. Hence, while the approximation consistently outperforms the lower bound in terms of both average and standard deviation of $\varepsilon$ (as revealed by the results in Table 2), the relative improvement of the approximation over the lower bound is most pronounced in balanced settings, where the average processing rates of both stages are of the same order of magnitude. 

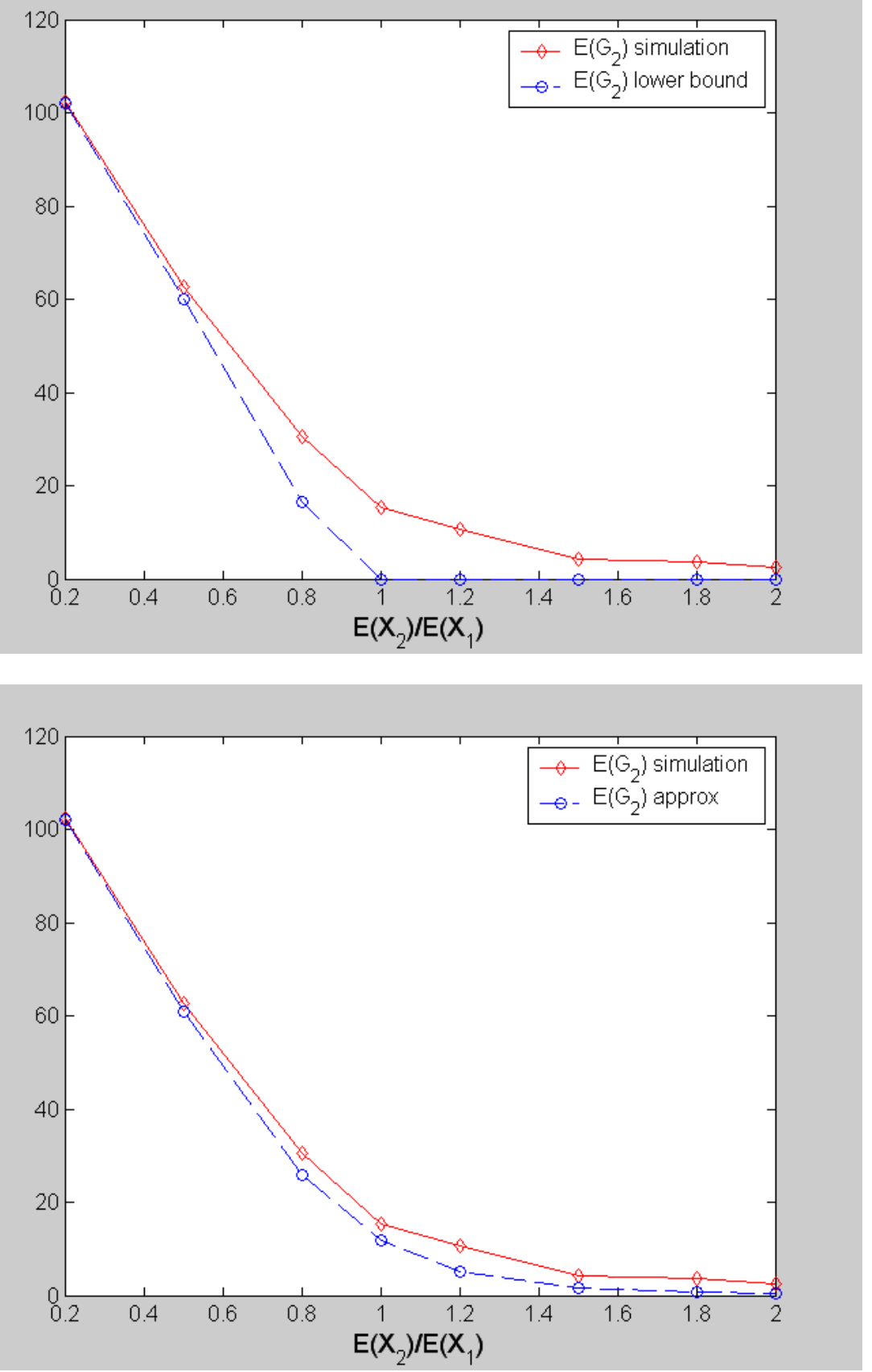

Figure 5. Comparison of the average value of $E\left[G_{2}\right]_{\text {sim }}$ versus the average value of $E\left[G_{2}\right]_{L B}$ (top pane), and versus the average value of $E\left[G_{2}\right]_{a p p}$ 


\section{Example}

Let's consider a two-stage flowshop where $N=30$ and $S_{1}=15$ (deterministic). The unit processing times on both stages are gamma distributed $\left(E\left(x_{1}\right)=10, E\left(x_{2}\right)=8, c_{x_{1}}^{2}=c_{x_{2}}^{2}=\right.$ $0.9)$, as is the setup time at stage $2\left(E\left(S_{2}\right)=0.5, c_{S_{2}}^{2}=0.9\right)$. As the process batch size equals 30 product units, the number of transfer batches in this setting may be equal to $T=1$ (in this case, no lot splitting is used), $T=2, T=3, T=5, T=6, T=10, T=15$ or $T=30$ (in this case, the process batch is split in the maximum number of sublots).

Figure 6 shows the average process batch makespan on stage 2 for this setting, in terms of $T$. The approximation is very close to the simulated value, at all values of $T$. An overview of the actual values and relative errors is given in Table 3.

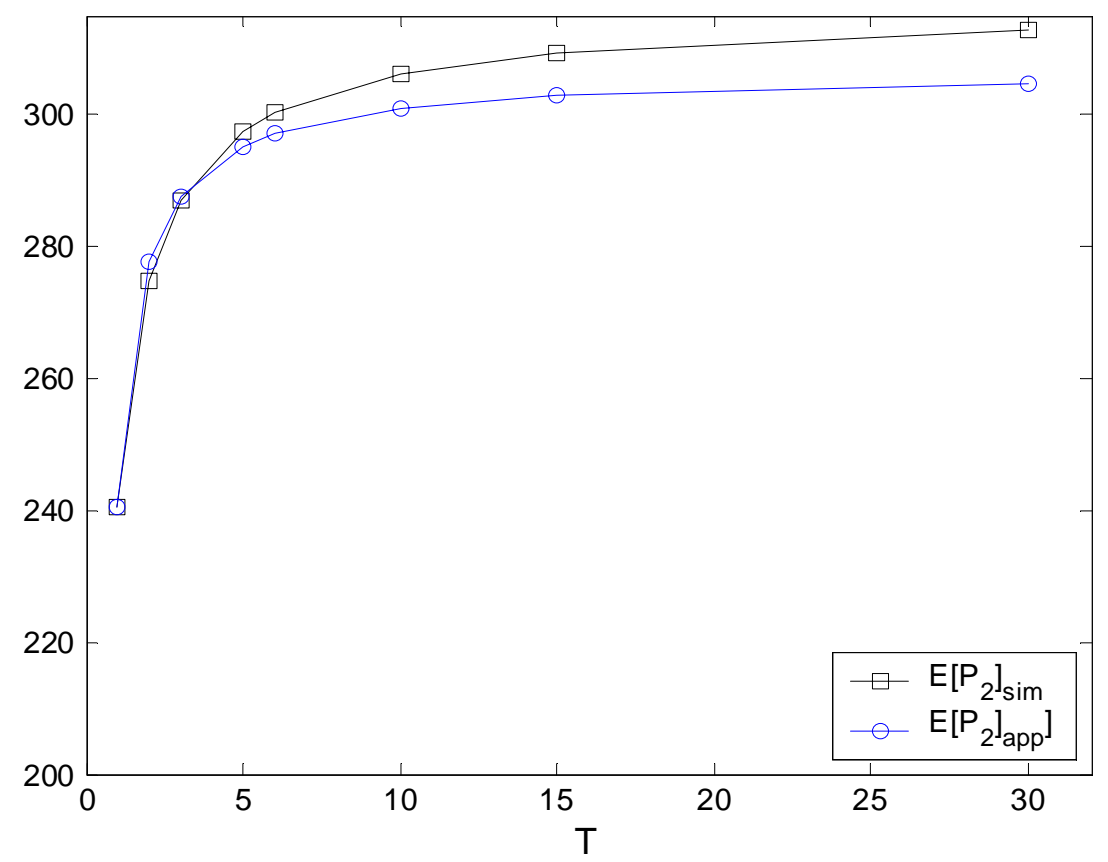

Figure 6. $E\left[P_{2}\right]_{a p p}$ versus $E\left[P_{2}\right]_{\text {sim }}$ for the example, in terms of $T$

Interestingly, the figure shows that the average process batch makespan on stage 2 increases as the process batch is split into a larger number of sublots. In fact, this will be the case whenever $E\left[x_{1}\right]>E\left[x_{2}\right]$. In that case, the average gap time on stage 2 will increase in T. A formal proof is presented in Appendix 2. This does not mean, however, that lot splitting should be avoided. Rather, it points towards a trade-off between the well-known improvement in flow time that can be obtained (which is due to the mechanism of overlap- 
Table 3. Performance of $E\left[P_{2}\right]_{\text {app }}$ compared to $E\left[P_{2}\right]_{\text {sim }}$, for the example

\begin{tabular}{l|lll}
$T$ & $E\left[P_{2}\right]_{\text {sim }}$ & $E\left[P_{2}\right]_{\text {app }}$ & $\varepsilon$ \\
\hline \hline 30 & 312.7170 & 304.8295 & $-2.52 \%$ \\
15 & 309.3135 & 302.9026 & $-2.07 \%$ \\
10 & 306.1598 & 300.9744 & $-1.69 \%$ \\
6 & 300.2889 & 297.1130 & $-1.06 \%$ \\
5 & 297.4579 & 295.1793 & $-0.77 \%$ \\
3 & 286.8907 & 287.4147 & $0.18 \%$ \\
2 & 274.7345 & 277.5953 & $1.04 \%$ \\
1 & 240.5100 & 240.5000 & $0.00 \%$ \\
\hline
\end{tabular}

ping operations, as described in section 1), and the gap time incurred on stage 2 . As the occurrence of gap times may imply a cost for the system, this observation emphasizes the importance of taking them into account when assessing the desirability of lot splitting in a stochastic setting.

\section{Conclusions}

In this paper, we have analyzed the occurrence of gap times in a two-stage stochastic flowshop with lot splitting. We have been able to derive a number of basic insights into the behavior of gap times, in terms of the system's characteristics (such as the difference in processing rates, the variability present in setup and processing times at the different stages, and the lot splitting policy used). Based upon these insights, we have developed a suitable approximation for the average process batch makespan on the second stage. This approximation explicitly takes into account the impact of setup and processing time variability on the occurrence of gap times, and hence outperforms the lower bound.

Though the occurrence of gap times is a major issue in stochastic settings, this paper provides (to the best of our knowledge) the first analysis of their behavior. In the future, we would like to extend the current model towards settings with more than one product type. Our results have also pointed towards a trade-off between the improvement in flow time obtained by using a lot splitting policy, and the occurrence of gap times caused by this policy. Hence, our interest also goes towards the development of an analytical model for estimating flow times in terms of the lot splitting policy, as this would enable us to include this trade-off in cost-benefit analyses. 


\section{Appendix 1: Proof for expression (4)}

Without loss of generality, we prove this equality for $T=5$. Note that $V_{i}$ in expression (3) equals

$$
V_{i}=\sum_{k=2}^{i} X_{1, k}-\left(W_{2}+S_{2}+\sum_{j=1}^{i-1}\left[X_{2, j}+g_{2}(j)\right]\right)
$$

such that we can write

$$
\begin{aligned}
G_{2}= & \sum_{i=2}^{T} \max \left[V_{i}, 0\right] \\
= & \underbrace{\max [Z(2), 0]}_{g_{2}(2)}+\underbrace{\max \left[Z(3)-g_{2}(2), 0\right]}_{g_{2}(3)}+\underbrace{\max \left[Z(4)-g_{2}(2)-g_{2}(3), 0\right]}_{g_{2}(4)} \\
& +\underbrace{\max \left[Z(5)-\sum_{t=2}^{4} g_{2}(t), 0\right]}_{g_{2}(5)}
\end{aligned}
$$

Moreover, we know that, for all random variables $X, A$ and $B$

$$
\max [X+A, X+B]=X+\max [A, B]
$$

By repeatedly applying this property, we get

$$
\begin{aligned}
g_{2}(2)+g_{2}(3) & =g_{2}(2)+\left[-g_{2}(2)+\max \left[Z(3), g_{2}(2)\right]\right] \\
& =\max [Z(3), \max [Z(2), 0]] \\
g_{2}(2)+g_{2}(3)+g_{2}(4) & =g_{2}(2)+g_{2}(3)+\left[-g_{2}(2)-g_{2}(3)+\max \left[Z(4), g_{2}(2)+g_{2}(3)\right]\right] \\
& =\max [Z(4), \max [Z(3), \max [Z(2), 0]]] \\
g_{2}(2)+g_{2}(3)+g_{2}(4)+g_{2}(5) & =\max [Z(5), \max [Z(4), \max [Z(3), \max [Z(2), 0]]]]
\end{aligned}
$$

Rewriting the last line, we get

$$
\begin{aligned}
G_{2} & =g_{2}(2)+g_{2}(3)+g_{2}(4)+g_{2}(5) \\
& =\max [Z(5), Z(4), Z(3), Z(2), 0]
\end{aligned}
$$

which is what we had to prove. 


\section{Appendix 2: Proof that $E\left(G_{2}\right)$ is increasing in $T$ when $E\left[x_{1}\right]>E\left[x_{2}\right]$}

From expression (7), we know that $E\left(G_{2}\right)$ can be approximated by

$$
E\left(G_{2}\right)=\frac{m_{V}}{2}+\frac{\operatorname{Exp}\left\{\frac{-m_{V}^{2}}{2 \sigma_{V}^{2}}\right\} \sigma_{V}^{2}}{\sqrt{2 \pi} \sigma_{V}}+\frac{m_{V}}{2} \operatorname{Erf}\left[\frac{m_{V}}{\sqrt{2} \sigma_{V}}\right]
$$

with $m_{V}$ and $\sigma_{V}^{2}$ given by

$$
\begin{aligned}
m_{V} & =(T-1) \frac{N}{T}\left[E\left(x_{1}\right)-E\left(x_{2}\right)\right]-E\left(S_{2}\right) \\
\sigma_{V}^{2} & =(T-1) \frac{N}{T}\left[\operatorname{Var}\left(x_{1}\right)+\operatorname{Var}\left(x_{2}\right)\right]+\operatorname{Var}\left(S_{2}\right)
\end{aligned}
$$

Assuming that $E\left(G_{2}\right)$ is continuous in $T$, the derivative of $E\left(G_{2}\right)$ towards $T$ equals

$$
\begin{aligned}
& \frac{\partial E\left(G_{2}\right)}{\partial T}=\frac{N}{4 T^{2}}[\underbrace{\operatorname{Exp}\left\{\frac{-m_{V}^{2}}{2 \sigma_{V}^{2}}\right\} \sqrt{\frac{2}{\pi}} \frac{\operatorname{Var}\left(x_{1}\right)+\operatorname{Var}\left(x_{2}\right)}{\sqrt{\frac{(T-1) N\left(\operatorname{Var}\left(x_{1}\right)+\operatorname{Var}\left(x_{2}\right)\right)}{T}+\operatorname{Var}\left(S_{2}\right)}}}_{>0} \\
& +2\left(E\left(x_{1}\right)-E\left(x_{2}\right)\right) \underbrace{\left.\left.1+\operatorname{Erf}\left[\frac{m_{V}}{\sqrt{2} \sigma_{V}}\right]\right)\right]}_{\geq 0}
\end{aligned}
$$

As the error function $\operatorname{Erf}[z]$ can only vary between -1 and $+1,(1+\operatorname{Erf}[z])$ is always larger than or equal to 0 , independent of $z$. Hence, we can conclude that

$$
E\left(x_{1}\right)-E\left(x_{2}\right)>0 \Longrightarrow \frac{\partial E\left(G_{2}\right)}{\partial T}>0
$$

In other words, when the average unit processing time on stage 1 exceeds the average unit processing time on stage 2 , the average gap time on stage 2 increases steadily when the process batch is split in a larger number of transfer batches. On the other hand, when $E\left(x_{1}\right)-E\left(x_{2}\right)<0$, it may happen that $\frac{\partial E\left(G_{2}\right)}{\partial T}$ becomes negative. In that case, the average gap time on stage 2 will decrease in terms of $T$.

\section{Acknowledgments}

This research was supported by the Research Foundation-Flanders (FWO-Vlaanderen). At the time of writing, Ms Van Nieuwenhuyse was a Postdoctoral Fellow of the Research Foundation-Flanders. 


\section{References}

Baker, K.R. and Jia,D. (1993) A comparative study of lot streaming techniques. Omega, 21, $561-566$.

Benjaafar, S. (1996) On production batches, transfer batches, and lead times. IIE Transactions, 28, 357-362.

Blumenfeld, D. (2001) Operations research calculations handbook, CRC Press, Boca Raton, FL.

Bozer, Y.A. and Kim, J. (1996) Determining transfer batch sizes in trip-based material handling systems. International Journal of Flexible Manufacturing Systems, 8, 313-356.

Bukchin, J., Tzur, M. and Jaffe, M. (2002) Lot splitting to minimize average flow time in a two-machine flow shop. IIE Transactions, 34, 953-970.

Bukchin, J. and Masin, M. (2004) Multi-objective lot splitting for a single product m-machine flowshop line. IIE Transactions, 36, 191-202.

Chen, J. and Steiner, G. (1996) Lot streaming with detached setups in three-machine flowshops. European Journal of Operational Research, 26, 591-611.

Chen, J. and Steiner, G. (1998) Lot streaming with attached setups in three-machine flowshops. IIE Transactions, 30, 1075-1084.

Cheng, T.C.E., Lin, B.M.T. and Toker, A. (2000) Makespan minimization in the two-machine flowshop batch scheduling problem. Naval Research Logistics, 47, 128-144.

Goldratt, E.M. and Cox, J. (1984) The Goal: a process of ongoing improvement, North River Press, New York.

Graves, S.C. and Kostreva, M.M. (1986) Overlapping operations in materials requirements planning. Journal of Operations Management, 6, 283-294.

Hopp, W.J., Spearman, M.L. and Woodruff, D.L. (1990) Practical strategies for lead time reduction. Manufacturing Review, 3, 78-84.

Hopp, W. J. and Spearman, M.L. (2000) Factory Physics: Foundations of Manufacturing Management, Irwin/McGraw-Hill, New York.

Jacobs, F.R. and Bragg, D.J. (1988) Repetitive lots: flow-time reductions through sequencing and dynamic batch sizing. Decision Sciences, 19, 281-294. 
Kalir, A.A. and Sarin, S.C. (2001) Optimal solutions for the single batch flow-shop lot streaming problem with equal sublots. Decision Sciences, 32, 387-397.

Kropp, D.H. and Smunt, T.L. (1990) Optimal and heuristic models for lot splitting in a flow shop. Decision Sciences, 21, 691-709.

Litchfield, J.L. and Narasimhan, R. (2000) Improving job shop performance through process queue management under transfer batching. Production and Operations Management, 9, 336-348.

Potts, C.N. and Kovalyov, M.Y. (2000) Scheduling with batching: a review. European Journal of Operational Research, 120, 228-249.

Ramasesh, R.V., Fu, H., Fong, D.K.H. and Hayya,J.C. (2000) Lot streaming in multistage production systems. International Journal of Production Economics, 66, 199-211.

Ross, S.M. (1996) Stochastic Processes, John Wiley and Sons Inc., New York.

Ruben, R.A. and Mahmoodi, F. (1998) Lot splitting in unbalanced production systems. Decision Sciences, 29, 921-949.

Santos, C. and Magazine, M. (1985) Batching in single-operations manufacturing systems. Operations Research Letters, 4, 99-103.

Sen, A., Topaloglu, E. and Benli, O.S. (1998) Optimal streaming of a single job in a two-stage flow shop. European of Operational Research, 110, 42-62.

Smunt, T.L., Buss, A.H. and Kropp, D.H. (1996) Lot Splitting in stochastic flow shop and job shop environments. Decision Sciences, 27, 215-238.

Suri, R. (1998) Quick Response Manufacturing: a companywide approach to reducing lead times, Productivity Press, Portland, OR.

Umble, M. and Srikanth, M.L. (1995) Synchronous Manufacturing: principles for world-class excellence, The Spectrum Publishing Company.

Van Nieuwenhuyse, I. 2004. Lot splitting in single-product flowshops: issues of delivery reliability, production disruptions and flow times. PhD thesis, Department of Applied Economics, University of Antwerp, Antwerp, Belgium.

Van Nieuwenhuyse, I. and Vandaele, N. (2004) Determining the optimal number of sublots in a single-product, deterministic flow shop with overlapping operations. International Journal of Production Economics, 92, 221-239. 
Wagner, B.J. and Ragatz, G.L. (1994) The impact of lot splitting on due date performance. Journal of Operations Management, 12, 13-25. 